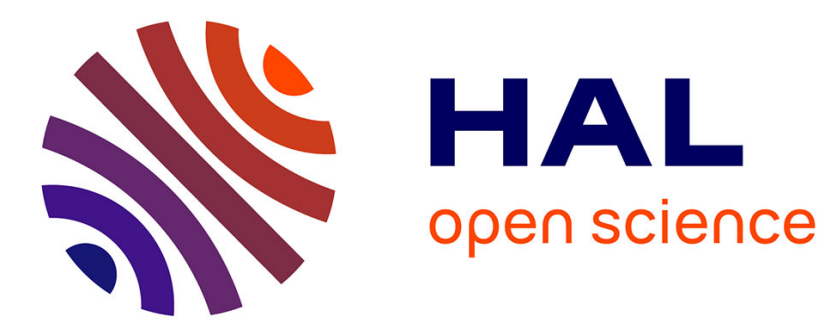

\title{
$\alpha$-Helix Unwinding as Force Buffer in Spectrins
}

Hirohide Takahashi, Felix Rico, Christophe Chipot, Simon Scheuring

\section{To cite this version:}

Hirohide Takahashi, Felix Rico, Christophe Chipot, Simon Scheuring. $\alpha$-Helix Unwinding as Force Buffer in Spectrins. ACS Nano, 2018, 12 (3), pp.2719-2727. 10.1021/acsnano.7b08973 . hal02106340

\section{HAL Id: hal-02106340 https://hal-amu.archives-ouvertes.fr/hal-02106340}

Submitted on 22 Apr 2019

HAL is a multi-disciplinary open access archive for the deposit and dissemination of scientific research documents, whether they are published or not. The documents may come from teaching and research institutions in France or abroad, or from public or private research centers.
L'archive ouverte pluridisciplinaire HAL, est destinée au dépôt et à la diffusion de documents scientifiques de niveau recherche, publiés ou non, émanant des établissements d'enseignement et de recherche français ou étrangers, des laboratoires publics ou privés. 


\title{
$\alpha$-HELIX UNWINDING AS VISCOUS FORCE
}

\section{BUFFER IN SPECTRIN}

\author{
Hirohide Takahashi ${ }^{1,2,3 \neq}$, Felix Rico ${ }^{1 \neq}$, Chris Chipot ${ }^{4} \&$ Simon Scheuring ${ }^{1,2,3 *}$ \\ ${ }^{1}$ U1006 INSERM, Université Aix-Marseille, Parc Scientifique et Technologique de Luminy, 163 avenue de \\ Luminy, 13009 Marseille, France \\ ${ }^{2}$ Department of Anesthesiology, Weill Cornell Medical College, 1300 York Ave, New York, NY 10065, USA \\ ${ }^{3}$ Departments of Physiology and Biophysics, Weill Cornell Medical College, 1300 York Ave, New York, NY \\ 10065, USA \\ ${ }^{4}$ Laboratoire International Associé Centre National de la Recherche Scientifique et University of Illinois at Urbana- \\ Champaign, UMR 7565, Université de Lorraine, B.P. 70239, 54506 Vandœuvre-lès-Nancy cedex, France and \\ Department of Physics, University of Illinois at Urbana-Champaign, 1110 West Green Street, Urbana, Illinois \\ 61801, USA \\ * Equal author contribution. \\ * Correspondence to: S. Scheuring, Email: sis2019@med.cornell.edu
}

\section{KEYWORDS}

AFM, Force Spectroscopy, High-Speed Force Spectroscopy, unfolding, unwinding

\begin{abstract}
Spectrins are cytoskeletal proteins located at the inner face of the plasma membrane, making connections between membrane anchors and the actin cortex, and between actin filaments. Spectrins share a common structure forming a bundle of 3 a-helices and play a major role during cell deformation. Here, we used high-speed force spectroscopy and steered molecular dynamics simulations to understand the mechanical stability of spectrin revealing a molecular force buffering function. We find that spectrin acts as a soft spring at short extensions (70-100 $)$. Under continuous external stretching, its $\alpha$-helices unwind leading to a viscous mechanical response over larger extensions (100-300 $\AA$ ), represented by a constant-force plateau in force/extension curves. This viscous force buffering emerges from a quasi-equilibrium competition between disruption and reformation of a-helical hydrogen bonds. Our results suggest
\end{abstract}


that, in contrast to $\beta$-sheet proteins which unfold in a catastrophic event, $\alpha$-helical spectrins dominantly unwind, providing a viscous force buffer over extensions about five times their folded length.

\section{INTRODUCTION}

Proteins have long been recognized to play either enzymatic or structural roles. More recently, protein mechanics has become of topical interest as biologists started to understand how mechanical cues are translated into not only mechanical but also biochemical responses ${ }^{12}$. As a consequence, many proteins are now considered to play dynamic mechanical roles. However, we still lack deeper understanding of how mechanical proteins function and what are their specificities.

Spectrin repeats are widely conserved in metazoan, being central in the cellular cytoskeleton ${ }^{3,4}$. Cytoskeletal filaments composed of spectrin repeats confer structural and mechanical stability to cells, notably by connecting the actin-myosin cortex to the plasma membrane, and by crosslinking actin filaments 5,6 . For example, the viscoelastic adaptation of red blood cells to rapid flow from wide arteries to narrow capillaries is compensated by the spectrin network 5, 7. Indeed, spectrin has been shown to unfold in red blood cells subjected to shear stress ${ }^{8}$. Protein families that contain spectrin repeats include alpha and beta spectrin, alpha-actinin, dystrophin and utrophin ${ }^{9-11}$. A spectrin repeat consists typically of about 110 amino acids and forms a three $\alpha$-helix bundle ${ }^{12}$ (Fig. 1a).

Atomic force microscopy (AFM) based force spectroscopy (FS) has revolutionized our understanding of the mechanical properties of proteins ${ }^{13,14}$. FS has been utilized to unfold and measure mechanical properties of spectrins ${ }^{15-18}$, revealing that they unfold following different pathways ${ }^{19}$, whereby individual $\alpha$-helixes interact with each other during unfolding ${ }^{17,}{ }^{18}$, and repeats exhibit mechanical cooperativity ${ }^{17}$. However, compared to $\beta$-fold proteins like titin ${ }^{13,20}$, the interpretation of FS data for spectrins has proven difficult, mainly due to the observed heterogeneity in the unfolding fingerprints at low forces $15,16,19,21$.

Steered molecular dynamics simulations (SMDS) - taking protein-solvent interactions into account - have been used to unfold proteins in silico and provided atomic descriptions of unfolding pathways ${ }^{22,23}$. SMDS have also been used to analyze spectrin mechanics, revealing multiple unfolding pathways, intermediate folding states and a weaker stability compared to $\beta$ rich domains ${ }^{21,24}$. However, typical SMDS for protein unfolding have been performed at high pulling speeds, in the range of $10^{-2}$ to $10^{2} \mathrm{~m} / \mathrm{s}$, and at $1 \mathrm{~m} / \mathrm{s}$ for spectrin ${ }^{25}$, rendering difficult the comparison with conventional FS data that unfolded proteins at typical velocities of $10^{-8}$ to $10^{-}$ ${ }^{5} \mathrm{~m} / \mathrm{s}$. To overcome this limitation, we recently introduced high-speed force spectroscopy (HSFS) as a unique tool to unfold proteins at velocities comparable to SMDS ${ }^{20}$. Using HS-FS, we now unfold proteins at velocities up to $10^{-2} \mathrm{~m} / \mathrm{s}$, accessible to SMDS, which, in the meantime, by dint of faster computers, progressed towards slower pulling speeds.

Here, we combine HS-FS and SMDS to unfold spectrin over almost seven orders of magnitude of velocity and with overlapping velocities between $10^{-3}$ and $10^{-2} \mathrm{~m} / \mathrm{s}$. Taking experimental and simulation data together, we suggest a mechanistic model of spectrin as a soft viscoelastic molecular system, where $\alpha$-helix unwinding takes a pivotal role as a long-range viscous force absorber. 


\section{RESULTS AND DISCUSSION}

For our unfolding experiments, we used the third spectrin repeat of alpha-actinin-4, because i) of its implication in stress-compensation between actin filaments and in actinmembrane anchoring ${ }^{26}$, ii) a high-resolution structure (PDB: 1WLX) is available, allowing SMDS of the exact same construct and straightforward comparison with experimental data to be performed, and iii) it does not contain cysteines, a potential source of complication due to intramolecular disulfide-bridge formation. The repeat comprises 129 amino acids. To perform HS-FS spectrin-unfolding, we prepared a chimeric protein construct: 4 spectrin repeats with XMod-dockerin-III 'helper domains', resulting in a surface-(spectrin)4-XMod-dockerin-III sequence. The latter domains serve for site-specific grabbing of the protein construct with a cohesin-III functionalized HS-AFM tip (Figure 1a). This setup has the elegance of covalent links to both the sample support and the tip ${ }^{27}$, and a well-defined interaction over the Xmoddockerin-III to CBM-cohesin-III complex (CBM stands for carbohydrate binding module). Furthermore, force curves feature a specific high-force unfolding and unbinding pattern allowing curve selection based on the dockerin-III/cohesin-III unfolding/unbinding signature and not on an "pre-defined" spectrin unfolding fingerprint (Figure 1b). The proper folding of the spectrin repeats in the chimeric protein was confirmed by circular dichroism (CD) (Supplementary Figure 1).

Using HS-FS, we unfolded spectrin at velocities ranging from $286 \mathrm{~nm} / \mathrm{s}\left(2.86 \times 10^{-7} \mathrm{~m} / \mathrm{s}\right)$ to $14.870 \mathrm{~mm} / \mathrm{s}\left(1.487 \times 10^{-2} \mathrm{~m} / \mathrm{s}\right)$, that is, over five orders of magnitude, thus, reaching the speed range of SMDS. We analyzed all force curves that contained the XMod-dockerin-III to cohesinIII unfolding/unbinding pattern, without discriminating by a putative or expected spectrinunfolding force-peak pattern (Figure 1b). Indeed, unlike studies performed on $\beta$-folded protein domains like titin ${ }^{15,20}$, the $\alpha$-helical fold of spectrin domains did not reproducibly reveal sawtooth-like unfolding patterns. In general, force-extension curves could be subdivided into two major unfolding events, namely events that displayed force peaks (Figure 1b, Figure 2a left) and events that displayed a rather flat force plateau (Figure 2a right).

At low velocities $(\leq 100 \mu \mathrm{m} / \mathrm{s})$, most force-extension curves exhibited a plateau force response of $\sim 25 \mathrm{pN}$ (Figure 2b). At higher retraction velocities $(\geq 100 \mu \mathrm{m} / \mathrm{s})$, individual peaks corresponding to domain unfolding events of individual spectrin repeats were observed more often (Figure 2b). In general, unfolding forces increased with increasing pulling velocity ${ }^{28,29}$, as can be seen in the raw-data force curves (Figure 2a left). The force values of individual spectrin unfolding peaks (Figure 2c left) and unfolding plateaus (Figure 2c right) were plotted against loading rate and pulling velocity, respectively, where filled symbols are the experimental data points and the open symbols the simulation data. The unfolding peak spectrum was well described using the BSK-model, an irreversible barrier crossing model valid over the widest dynamic range applied ${ }^{30}$, while the force plateau spectrum agreed with a near-equilibrium model where unfolding and refolding are possible (FNdY model) ${ }^{31}$. These interpretations are justified because force peaks are signatures of catastrophic unfolding events and refolding is unlikely. Conversely, the force plateaus represent a velocity-independent force response at low velocities, in agreement with possible refolding of the $\alpha$-helical turns. Both datasets were well fitted by the respective models. The BSK fitting indicated that the transition from the Bell-Evans regime (where proteins unfold stochastically as a function of the rate at which the force is applied) to the so-called deterministic regime (where proteins unfold faster than their ability to explore their 
conformational space) occurs at a loading rate of $\sim 2 \times 10^{10} \mathrm{pN} / \mathrm{s}$ and a critical force of $\sim 285 \mathrm{pN}$, comprised within the SMDS regime. The BSK fit yields an energy landscape in which the unfolding transition barrier is located at $x_{\mathrm{b}}=0.53 \pm 0.03 \mathrm{~nm}$, a diffusion coefficient $D_{m}=(3.4 \pm 0.6) \times 10^{6} \mathrm{~nm}^{2} \mathrm{~s}^{-1}$ and the barrier height is $\Delta G=18 \pm 1 \mathrm{kB} T$, leading to a unfolding rate at zero force $k^{0}=11 \mathrm{~s}^{-1}$ and a molecular stiffness $k_{\mathrm{m}}=543 \mathrm{pN} / \mathrm{nm}$. In comparison, the $\beta$-fold protein core of titin I-91, which we have unfolded previously ${ }^{20}$, revealed an unfolding barrier distance $x_{\mathrm{b}}=0.89 \mathrm{~nm}$ and a height of $\Delta G=36 k_{\mathrm{B}} T$, with a $k^{0} \sim 10^{-10} \mathrm{~s}^{-1}$, thus a much more stable fold that only unfolded at much higher forces. The fast unfolding rate at zero force of spectrin $\left(k^{0}=11 \mathrm{~s}^{-1}\right)$ explains the low probability of observing unfolding peaks at low velocities (Fig. 2B), suggesting that the $\alpha$-helical spectrin repeats are much more compliant and labile structures than $\beta$-folds.

The contour length difference between unfolding peaks was obtained from WLC fits. The distribution showed a broad peak at $\sim 34 \mathrm{~nm}$, with minor peaks at $\sim 10 \mathrm{~nm}$ and $\sim 50 \mathrm{~nm}$ (Supplementary Figure 2). The unfolded length of one spectrin repeat is $\sim 49 \mathrm{~nm}$ (assuming a stretch of $\sim 0.38 \mathrm{~nm}$ for each of the 129 amino acids in a repeat) and the length of the folded oriented structure is $\sim 7 \mathrm{~nm}$ (distance between N-and C-terminus) (Supplementary videos 1 to 5). This trimodal distribution suggests that spectrin unfolds through different intermediate states, one via the release of 26 residues ( $\sim 7$ helix turns) leading to a $10 \mathrm{~nm}$ extension, another via 89 residues $(\sim 25$ helix turns, $34 \mathrm{~nm})$ and a third one involving the whole spectrin repeat. Our SMDS provide a mechanistic description of this multiple pathways as described below.

While some force-extension profiles exhibited one or more force peaks for the spectrinrepeats unfolding, extended plateau regions were almost always present. Some curves showed a plateau over the entire spectrin-repeats extension (Fig. 2a, right). Thus, the plateau response appeared to be the most relevant unfolding pathway, especially at slow $(<100 \mu \mathrm{m} / \mathrm{s})$ velocities (Fig. 2b). Interestingly, in this velocity regime, the plateau force values did not reveal any velocity dependence (Figure 2c, right). However, beyond this critical velocity $(100 \mu \mathrm{m} / \mathrm{s})$, the plateau force scaled logarithmically with the rate of loading, suggesting a thermally activated (Bell-Evans) regime. We interpret the constant dynamic response at low velocity as a nearequilibrium process, where refolding dominates over unfolding, as described by the FNdY model 31. A similar interpretation is a two-state model with a short distance to the transition state, as described before for polysaccharides undergoing conformational transition upon stretch ${ }^{32}$. The FNdY model fit of the plateau dynamic force spectrum results in a distance to the transition barrier $x_{\mathrm{t}}=0.18 \pm 0.01 \mathrm{~nm}$, and very fast intrinsic (zero-force) unfolding and refolding rates $k^{0} \mathrm{NI}=(6.7 \pm 1.0) \times 10^{6} \mathrm{~s}^{-1}$ and $k_{\mathrm{NI}}^{0}=(7.8 \pm 1.2) \times 10^{6} \mathrm{~s}^{-1}$, respectively. Once the pulling rate becomes faster than the refolding rate, the system enters into the Bell-Evans regime. This model fits not only well the data, but it also agrees nicely with the observed phenomenon, i.e. a viscous response with a constant force value of a continuously unwinding protein. Moreover, the fitted distance to the unfolding barrier $x_{\mathrm{t}}=0.18 \mathrm{~nm}$ is similar to that of hydrogen bonds in $\alpha$-helices. Without a catastrophic unfolding event, but following successive unwinding of its helices, it can be anticipated that helix turns transiently reform under slow pulling velocity. Such a refolding of the $\alpha$-helices was, indeed, observed in the simulations as described below.

To gain atomic-scale insight into the unfolding process, we performed constant-velocity SMDS of the very same spectrin domain ( $1 \mathrm{WLX})$ at pulling rates covering 2.5 orders of magnitude from $5.0 \times 10^{-3} \mathrm{~m} / \mathrm{s}$ to $1.0 \mathrm{~m} / \mathrm{s}$. We performed a total of $10 \mathrm{SMDS}$ and all results show the following behavior: (i) while unfolding forces increase with pulling velocity, there is no conserved and recognizable force-extension pattern (Figure 3a); (ii) the total number of $\alpha$ - 
helical amino-acid residues in the spectrin repeat decreases quasi-linearly with 1 amino-acid per $0.3 \mathrm{~nm}$ additional extension, in agreement with a continuous unwinding of helices. The expected barrier to cross from folded and unfolded helical states is expected to be around half this extension $(0.15 \mathrm{~nm})$, which is similar to twice the fitted value of $0.18 \mathrm{~nm}$ from HS-FS experiments (Figure 3b); (iii) helix-3 (C-terminal helix) unfolds first, prior to helix-1 (Nterminal helix). The central helix-2 is the most robust and long-lived, preserving its secondary structure until about $30 \mathrm{~nm}$ extension. This is a direct consequence of the down-up-down orientation of the three helices in the tertiary structure of the spectrin repeat. Helix-2, which is oriented in opposite direction with respect to the pulling force vector, experiences stretching forces only after the unwinding of helices- 1 and -3 (Figure 3c). (iv) As a consequence of the short lifetime and low stretch resistance of helix-3, all interactions with helix-3 vanish first. Accordingly, there is a propensity for a force peak at about $10 \mathrm{~nm}$ extension when helix -3 vanishes and all helices-2-3 and helices-1-3 contacts are lost (Figure 3d). This phenomenon is more pronounced at higher velocities, in line with the higher probability of finding unfolding peaks in faster pulling experiments (Figure 2b). We, therefore, propose that the interactions with helix-3 provide an elastic response, at short distance and loss of the interhelical contacts leads to collapse of the tertiary fold and force-peak observation (Figure 3a, $\leq 10 \mathrm{~nm}$ ). The helices-1-2 interactions are the longest lived and the loss of interhelical contacts may be responsible for the experimentally observed peak distribution at $\sim 30 \mathrm{~nm}$. However, continuous unwinding of the helices results in a force plateau and provides a viscous response over large extension that may function as a force buffer in vivo (Figure 3a, extension $\leq 30 \mathrm{~nm}$ ).

SMDS movies at different unfolding velocities (Supplementary videos 1 to 4) and snapshots of the trajectories illustrate the key events of the spectrin force response (Figure 4a): The fully folded spectrin repeat first orients along the pulling vector without the application of a measurable force and the protein possesses an end-to-end distance of $\sim 7 \mathrm{~nm}$ (state 1). Helix-3 unwinds first (state 2) up to an extension of $\sim 15 \mathrm{~nm}$ (state 3 ). Once helix-3 is completely unwound a major reorganization of helix-2 occurs, typically reorienting it from antiparallel to parallel with respect to the extension (state 4). Finally, helix-1 and helix-2 unwind to an extension of $\sim 30 \mathrm{~nm}$ (state 5). It is noteworthy that in the slowest SMDS, unwinding and reformation of $\alpha$-helical amino acids are observed several times within $\sim 0.1 \mu$ s (Figure $4 \mathbf{b}$ ). This finding is a hallmark of the viscous force buffer represented by the $\alpha$-helices in spectrin and supports our interpretation of a near-equilibrium regime at low velocities, in which winding and unwinding rates compete. The applied forces when these events occurred were $100 \mathrm{pN}$ (winding) and $150 \mathrm{pN}$ (unwinding), respectively. The unwinding and rewinding rates from the plateau force spectrum at such loads are expected to be $6.4 \times 10^{7} \mathrm{~s}^{-1}$ and $7.8 \times 10^{6} \mathrm{~s}^{-1}(F=100 \mathrm{pN})$ and $5.9 \times 10^{8} \mathrm{~s}^{-1}$ and $4.2 \times 10^{6} \mathrm{~s}^{-1}(F=150 \mathrm{pN})$, in good agreement with the unfolding and refolding events observed in SMDS within $0.08 \mu$ s and $0.11 \mu$ s (thus at rates around $10^{7} \mathrm{~s}^{-1}$ ).

Altogether, we consolidate HS-FS as a unique tool for the characterization of protein mechanics over the widest dynamic range, allowing combination with SMDS at overlapping unfolding velocities. Furthermore, an atomistic understanding of the protein mechanics is gained. We show that spectrin is a viscoelastic molecular force buffer, occasionally acting as a soft spring at short extensions, followed by a long-distance viscous response dominated by the unwinding of $\alpha$-helices. One can hypothesize that the adaptation of a red blood cell to extended duration of squeezing in microcapillaries or the spleen is better served by a viscous force buffer than by an elastic one. An elastic spring would work against the imposed compression and potentially lead to blockage of a red blood cell during passage, while the viscous adaptation 
would comply with extended copaction. In summary, we provide evidence that unwinding of $\alpha$ helices are at the basis of a viscous force response in $\alpha$-helical mechanical proteins, obeying a novel, reversible helix unwinding/winding force buffer mechanism.

\section{MATERIALS AND METHODS}

\section{Preparation of DNA construct for spectrin concatamer}

To prepare the DNA construct, we first modified the pET28a DNA plasmid as follows: The entire pET28a was digested with Nco I and Bam HI (New England Biolab) and run on a 1\% agarose gel. A single band appearing at $\sim 5.3 \mathrm{~kb}$ was extracted and purified with QIA gel extraction kit (QIAGEN). The linearized cDNA fragment was ligated to a cDNA sequence, which was prepared by annealing two DNA oligos: 5'CATGGGCCATCATCATCATCATCATCATCATCATCATCATG3' and 5'GATCCATGATGATGATGATGATGATGATGATGATGGCC3'. The prepared DNA plasmid was named pET28a (BamHI-Hisx10-HindIII). The cDNA sequence coding for the spectrin repeat (1WLX) was amplified by thermal cycler with two DNA primers: 5' CGTAGGATCCACAGAGAAGCAGCTGGAGGCCATCG3' and GCTAGATCTTCCTCCCTGCTGCTTGCTCTGCTCCTCC3'. For DNA amplification the thermal cycle started with $95^{\circ} \mathrm{C}$ for 5 minutes, followed by repeating 32 cycles of the following 3 steps: $95^{\circ} \mathrm{C}$ for 30 seconds, $63^{\circ} \mathrm{C}$ for 30 seconds, $72^{\circ} \mathrm{C}$ for 1 minute, ending with $72^{\circ} \mathrm{C}$ for 5 minutes. The amplified DNA was run on a $1 \%$ agarose gel with constant voltage at $100 \mathrm{~V}$. The band around 400 bps was collected and purified with QIA gel extraction kit. The purified DNA fragment was then digested with restriction enzymes Bam HI and Bgl II (New England Biolab), then again run on a $1 \%$ agarose gel and purified using the same procedure. The digested DNA fragment was inserted into the pET28a (BamHI-Hisx10-HindIII) to give a pET28a-spectrin1 DNA construct. The DNA sequence of the spectrin repeat was confirmed by Sanger DNA sequencing. The DNA plasmid (pET28a-spectrin1) was then linearized by BamHI, and the fragment coding spectrin (1WLX) with ends BamHI(5') and Bgl II(3') was inserted to give a pET28a-spectrin2 DNA construct. In order to prepare a concatamer of 4 spectrin repeats the DNA fragment coding for the 2 spectrin repeats was cut out from the pET28a-spectrin2 DNA construct, inserted into the pET28a-spctrin2 DNA fragment which was linearized by BamHI to give a pET28a-spectrin4 DNA construct. The DNA sequence corresponding to the concatamer of 4 spectrin repeats was confirmed by Sanger sequencing.

\section{Preparation of DNA construct for spectrin-XMod-dockerin chimera}

In order to make a chimera of spectrins with ybbR and XMod and dockerin-III, first a short DNA fragment was prepared by annealing two cDNA the following two oligos 5'GATCTGACTCTCTGGAATTCATCGCTTCTAAACTGGCTGGATCCAAGGTACCATGC C3'

5'TCGAGGCATGGTACCTTGGATCCAGCCAGTTTAGAAGCGATGAATTCCAGAGAGT CA3', and inserted into the pET28a-spectrin4 plasmid, which was linearized by BamHI. We then inserted the DNA fragment coding XMod and dockerin-III, which was cut out by digestion with KpnI and XhoI (New England Biolab). The DNA sequence corresponding to the amino acid sequences of ybbR, 4 spectrin repeats, XMod-dockerin-III (from N-terminus to C-terminus) was confirmed by Sanger sequencing. 


\section{Protein purification}

The DNA plasmid coding 4 repeats of ybbR-spectrin and XMod-dockerin-III was transformed into BL21 (DE3) cells. The BL21 cells were initially inoculated in $50 \mathrm{ml}$ of LB medium containing $50 \mathrm{ng} / \mathrm{ml}$ kanamycin and incubated overnight at $37^{\circ} \mathrm{C}$, with shaking. Next day, the medium was transferred into $1 \mathrm{~L}$ of fresh LB medium containing $100 \mathrm{ng} / \mathrm{ml}$ kanamycin and incubated at $37^{\circ} \mathrm{C}$ with shaking until the $\mathrm{OD}_{600}$ value reached at 0.6. To induce protein expression, isopropyl $\beta$-D-1-thiogalactopyranoside (IPTG) was added to the medium and incubated at $20^{\circ} \mathrm{C}$ with shaking overnight. Next day, the resulting medium was centrifuged at $5,000 \mathrm{x} \mathrm{g}$ for 10 minutes and the pellet collected. The pellet was resuspended in $10 \mathrm{~mL}$ of HEPES-buffered saline (20mM HEPES-NaOH, pH 7.5, 100mM NaOH). After addition of $1 \mathrm{mM}$ phenylmethylsulfonyl fluoride (PMSF), the bacterial debris was sonicated using a probe sonicator on ice (3 times for 20 seconds with 30 seconds interval). Triton X-100, DNase and RNase were added into the suspension at $0.1 \%, 10 \mu \mathrm{g} / \mathrm{ml}, 20 \mu \mathrm{g} / \mathrm{ml}$ final concentrations, respectively, and incubated for 30 minutes at $4{ }^{\circ} \mathrm{C}$ with gentle shaking. The resulting solution was centrifuged at $10,000 \times \mathrm{g}$ for 30 minutes and the supernatant was mixed with $1 \mathrm{~mL}$ of NTAnickel resin, which was equilibrated with HEPES-buffered saline. The mixture was incubated for 1 hour at $4^{\circ} \mathrm{C}$ with gentle shaking. The resin was then rinsed following: centrifugation at $100 \mathrm{xg}$ for 1 minute, removal of the supernatant and resuspension in $100 \mathrm{mM}$ of imidazole-HCl, $\mathrm{pH} 7.5$. The rinsing process was repeated 3 times. The final purified fraction was eluted in $2 \mathrm{~mL}$ of $250 \mathrm{mM}$ of imidazole-HCl. To remove imidazole, the eluate was dialyzed against $1 \mathrm{~L}$ of HEPESbuffered saline at $4^{\circ} \mathrm{C}$ overnight with gentle stirring.

\section{Protein immobilization on the glass surface:}

In order to immobilize protein on the surface of glass, we first cleaned glass rods $(1.5 \mathrm{~mm}$ diameter, 2.0mm height) with a plasma cleaner (Plasma cleaner Zepto, Diener electronic) using $80 \%$ output for 5 minutes at 0.3 mbar under oxygen gas. The cleaned glass rods were rinsed in analytical grade ethanol (more than $99.9 \%$ purity, Sigma), and incubated in 5\% 3(aminopropyl)triethoxysilane in ethanol for 10 minutes at room temperature. The treated glass rods were then rinsed with ethanol and baked at $80^{\circ} \mathrm{C}$ for 30 minutes. To deprotonated the functionalized amines, the glass rods were soaked in $20 \mathrm{mM} \mathrm{Na} \mathrm{HPO}_{4}$ overnight. To functionalize maleimide groups, the glass rods were incubated in 5mM NHS-PEG(27)-Maleimide (Polypure) in phosphate-buffered saline (PBS: $10 \mathrm{mM} \mathrm{Na}_{2} \mathrm{HPO}_{4}, 1.76 \mathrm{mM} \mathrm{KH}_{2} \mathrm{PO}_{4}, 137 \mathrm{mM}$ $\mathrm{NaCl}, 2.7 \mathrm{mM} \mathrm{KCl}$ ) for 1 hour at room temperature. The glass rods were then incubated in $20 \mathrm{mM}$ Coenzyme A trilithium salt (Sigma) for 1 hour at room temperature. After rinsing with Milli-Q water, the glass rods were functionalized with the protein by incubation with $100 \mu \mathrm{g} / \mathrm{mL} \mathrm{ybbR}-4$ $\mathrm{x}$ spectrin-XMod-dockerin in presence of $1 \mu \mathrm{M}$ sfp in $25 \mathrm{mM}$ Tris- $\mathrm{HCl}, \mathrm{pH} 7.2,75 \mathrm{mM} \mathrm{NaCl}$, $1 \mathrm{mM} \mathrm{CaCl}_{2}$ and $1 \mathrm{mM} \mathrm{MgCl} 2$ for 1 hour at room temperature. The protein covered glass rods were then immediately used for measurements.

\section{Tip functionalization:}

Cantilevers (BL-AC10DS, Olympus) were cleaned in the plasma cleaner and functionalized with coenzyme A following the same procedure as described in the section above "Protein immobilization on the glass surface". The cantilevers were then functionalized with protein by incubation in $100 \mu \mathrm{g} / \mathrm{mL}$ ybbR-cohesin-III in presence of $1 \mu \mathrm{M}$ of sfp in $25 \mathrm{mM}$ Tris$\mathrm{HCl}, \mathrm{pH} 7.2,75 \mathrm{mM} \mathrm{NaCl}, 1 \mathrm{mM} \mathrm{CaCl} 2$ and $1 \mathrm{mM} \mathrm{MgCl}_{2}$ for 1 hour at room temperature. The cantilevers were then immediately used for measurements. 


\section{High-speed force spectroscopy (HS-FS):}

The force measurement was performed as described before ${ }^{20}$. Briefly, the high-speed force spectroscopy setup is based on a SS-NEX HS-AFM (RIBM, Japan) (16). ${ }^{33}$ The deflection sensitivity and spring constant of the cantilevers were determined before every experiment using the thermal fluctuations method ${ }^{34}$. The cantilevers (BL-AC10DS) had a typical resonance frequencies of $1.3 \mathrm{MHz}$ and $0.6 \mathrm{MHz}$ in air and liquid, respectively. The HS-FS setup was controlled with home-built software using a multichannel analog to digital converter with maximum acquisition rate of 20 megasamples per second and channel, to drive piezo displacement and acquire cantilever deflection (LabView programming, PXI-5122 card, National Instruments, USA). The spring constant of the cantilevers was determined using the Sader method applying the correction for higher modes corresponding to rectangular cantilevers ${ }^{33}$. The sensitivity of the photodiode was extracted from the thermal noise spectrum using the calibrated spring constant ${ }^{34-36}$. Force spectroscopy measurements were performed in $25 \mathrm{mM}$ Tris-HCl, $\mathrm{pH} 7.5,75 \mathrm{mM} \mathrm{NaCl}$ and $2 \mathrm{mM} \mathrm{CaCl} 2$ by approaching the sample surface to the cantilever at $\sim 1 \mu \mathrm{m} / \mathrm{s}$ velocity. The contact was maintained for 1 second, followed by retraction of the surface at velocities ranging from $0.286 \mu \mathrm{m} / \mathrm{s}$ to $14870 \mu \mathrm{m} / \mathrm{s}$.

\section{Force-extension curve analysis:}

The protein construct used in this work contains four concatenated spectrin repeats followed by an XMod and a dockerin-III domain. Dockerin specifically binds to colhesin-III functionalized on the cantilever and the cohesion-dockerin unbinding event generates a specific, high force peak (more than $200-300 \mathrm{pN}$, sometimes preceded by an unfolding peak with contour length $50-80 \mathrm{~nm}$ ) ${ }^{27}$. We therefore collected all force curves with a cohesion-dockerin unbinding force peak as specific force-distance curves considered for analysis. We selected 10-30 successful force curves at each pulling speed to analyze forced unfolding events. The force distance curves, which contained dockerin-cohesin unbinding events were categorized to peaks and plateaus. Peak detection was carried out using a home-built semiautomatic procedure (written in Matlab, Mathworks) based on a threshold defined on the first derivative of the deflection signal.

\section{Fitting of the dynamic force spectra:}

\section{BSK model: complete unfolding}

The dynamic force spectrum of complete unfolding (peaks) was interpreted in terms of the BSK model for rapid force spectroscopy, which is valid over the wide range of pulling rates applied in HS-FS and SMDS ${ }^{30}$. The BSK model describes two loading rate $\left(r_{f}\right)$ regimes of unfolding forces: a logarithmic regime at low loading rates, and square root regime at high loading rates, that can be approximated as

$$
F \approx \frac{k_{B} T}{x_{\beta}} \ln \left[1+\frac{r_{f} x_{\beta}}{k^{0} k_{B} T} e^{-\gamma-\frac{\Delta G^{\ddagger}}{k_{B} T}}\right]+\sqrt{r_{f} \frac{2 x_{\beta} k_{B} T}{D_{m}}}
$$

being $\gamma=0.5772$ the Euler constant, $k_{\mathrm{B}} T$, the thermal energy, $D_{\mathrm{m}}$, the intrinsic diffusion constant, $x_{\beta}$, the distance to the transition state, and $\Delta G^{\ddagger}$, the barrier height. The intrinsic unfolding rate $\left(k^{0}\right)$ is defined by the Kramers rate for a harmonic potential 


$$
k^{0}=\frac{2 D_{m} \Delta G^{\ddagger}}{x_{\beta}^{2} k_{B} T} \sqrt{\frac{\Delta G^{\ddagger}}{\pi k_{B} T}} e^{-\frac{\Delta G^{\ddagger}}{\pi k_{B} T}}
$$

The BSK fit of the unfolding peaks spectrum resulted in $D_{\mathrm{m}}=(3.4 \pm 0.6) \times 10^{6} \mathrm{~nm}^{2} / \mathrm{s}$, $x_{\beta}=0.53 \pm 0.03 \mathrm{~nm}$, and $\Delta G^{*}=18.4 \pm 0.4 \mathrm{kB} T$. Leading to an intrinsic unfolding rate of $k^{0}=11.4 \mathrm{~s}^{-1}$, and a molecular stiffness $k_{\mathrm{m}}=\frac{2 \Delta G^{\ddagger}}{x_{\beta}^{2}}=543 \mathrm{pN} / \mathrm{nm}$ and critical force $F_{\mathrm{c}}=285 \mathrm{pN}$.

FNdY model: $\alpha$-helix unwinding

The $\alpha$-helix unwinding forces were characterized by a "plateau" during the stretching regime. The average unwinding force versus loading rate was fitted using a reversible binding model recently developed by Friddle et al. ${ }^{31}$ (FNdY model). In this model, the system has two dynamic regimes: a near-equilibrium regime with unfolding rates slower than refolding rates that results in a force plateau in the dynamic force spectrum, and a non-equilibrium, kinetic regime at fast unfolding. The average force is described by

$$
F=f_{e q}+\frac{k_{B} T}{x_{t}} \ln \left[1+e^{-g} \frac{x_{t} l_{r}}{k_{N I}\left(f_{e q}\right) k_{B} T}\right]
$$

where $\gamma=0.5772$ is the Euler constant, $x_{t}$ is the distance to the transition state, $f_{e q}$ is the equilibrium force and the loading rate $l_{\mathrm{r}}=k_{m} v$ is determined from the spring constant $\left(k_{\mathrm{m}}\right)$ of the folded molecule $\left(k_{m} \sim 543 \mathrm{pN} / \mathrm{nm}\right.$ as determined experimentally from the fit of the dynamic force spectrum described above) and the pulling velocity $(v) . k_{\mathrm{NI}}\left(f_{\mathrm{eq}}\right)$ is the rate of unfolding from the native to the intermediate state.

We assume that the energy barrier of unwinding is flanked by the complete unfolding energy landscape with a spring constant estimated from the BSK fit described above. Thus, for spectrin unwinding, refolding originates from the stiff molecular spring constant $(\sim 543 \mathrm{pN} / \mathrm{nm})$ and not from the much softer spring constant of the cantilever and the unfolded polypeptide $(\sim 4 \mathrm{pN} / \mathrm{nm})$. Thus, the unfolding rate, $k \mathrm{NI}(f)$, is described for any force $f$ as

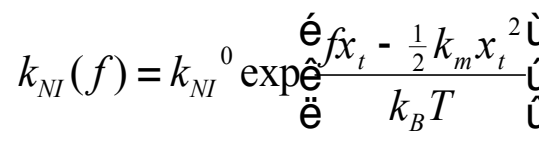

(Eq. 4)

where $k_{N I}{ }^{0}$ is the intrinsic unfolding rate and $k_{m}$ is the molecular spring constant of the energy potential defined above.

According to this model, at low loading rates, the dynamic force spectrum, tends to the equilibrium force defined as

$$
f_{e q}=\sqrt{2 k_{m} \Delta G_{N I}}
$$

being $\Delta G_{N I}=G_{I}-G_{N}$ the equilibrium free energy of the native state relative to the intermediate unwinding state. The refolding rate from the intermediate to the native state is given by

$$
k_{I N}(f)=k_{N I}(f) \exp \left[\frac{\mathrm{D} G_{N I}-\frac{f^{2}}{2 k_{m}}}{k_{B} T}\right]
$$


This model was fitted to the average plateau forces versus pulling velocity using a nonlinear least squares method by Chi-squared minimization using the standard error of the mean as weighting. The fit lead to $f_{\mathrm{eq}}=26 \pm 2 \mathrm{pN}, x_{\mathrm{t}}=0.18 \pm 0.01 \mathrm{~nm}$, and $k_{\mathrm{NI}}^{0}=(6.7 \pm 1.0) \times 10^{6} \mathrm{~s}^{-1}$, resulting in $\Delta G_{N I}=0.54 k_{\mathrm{B}} T$ and $k_{\mathrm{IN}}^{0}=7.8 \times 10^{6} \mathrm{~s}^{-1}$.

\section{Steered molecular dynamics simulations (SMDS):}

The molecular assembly consisted of one spectrin repeat hydrated by 62,369 water molecules, which corresponds to 189,175 atoms and to a simulation cell of dimensions equal to $60 \times 60 \times 520 \AA^{3}$. For the simulation at the slowest pulling speed, corresponding to smaller extension, a smaller assay was employed, with 21,993 water molecules and a cell of dimensions equal to $60 \times 60 \times 186 \AA^{3}$ containing a total of 68,135 atoms. All the simulations presented herein were performed employing the scalable program NAMD $2.11^{37}$. The CHARMM36 force field ${ }^{38}$ was used to describe spectrin and its aqueous medium. The r-RESPA multiple time-step algorithm ${ }^{39}$ was employed to integrate the equations of motion with a time step of 1 and 2 fs for short- and long-range interactions, respectively. Covalent bonds involving hydrogen atoms were constrained to their equilibrium length by means of the SHAKE/RATTLE ${ }^{40}$ and SETTLE ${ }^{41}$ algorithms. The temperature and the pressure were maintained at $300 \mathrm{~K}$ and $1 \mathrm{~atm}$, respectively, using Langevin dynamics and the Langevin piston method ${ }^{42}$. Long-range electrostatic forces were taken into account by means of the particle mesh Ewald algorithm. A $12 \AA$ cutoff was introduced to truncate van der Waals and short-range Coulombic interactions. Periodic boundary conditions were applied in the three directions of Cartesian space. In SMDS, the position of the $\mathrm{N}$-terminal amino acid residue was tethered, while the center of mass of the C-terminal amino acid residue was harmonically restrained with a force constant of $200 \mathrm{pN} \mathrm{nm}^{-1}$ to move at constant velocity along the z-direction of Cartesian space. For each pulling speed of $1.00 \mathrm{~m} \mathrm{~s}^{-1}$, $0.10 \mathrm{~m} \mathrm{~s}^{-1}$ and $0.05 \mathrm{~m} \mathrm{~s}^{-1}$, three independent simulations were run, covering about $300 \AA$ of extension. An additional SMDS was performed at $0.005 \mathrm{~m} \mathrm{~s}^{-1}$ over a shorter extension of about $100 \AA$. The aggregate time for these ten SMDS amounted to $4.69 \mu$ s. Visualization and analyses of the SMDS trajectories were performed with the VMD program ${ }^{43}$. 
FIGURES

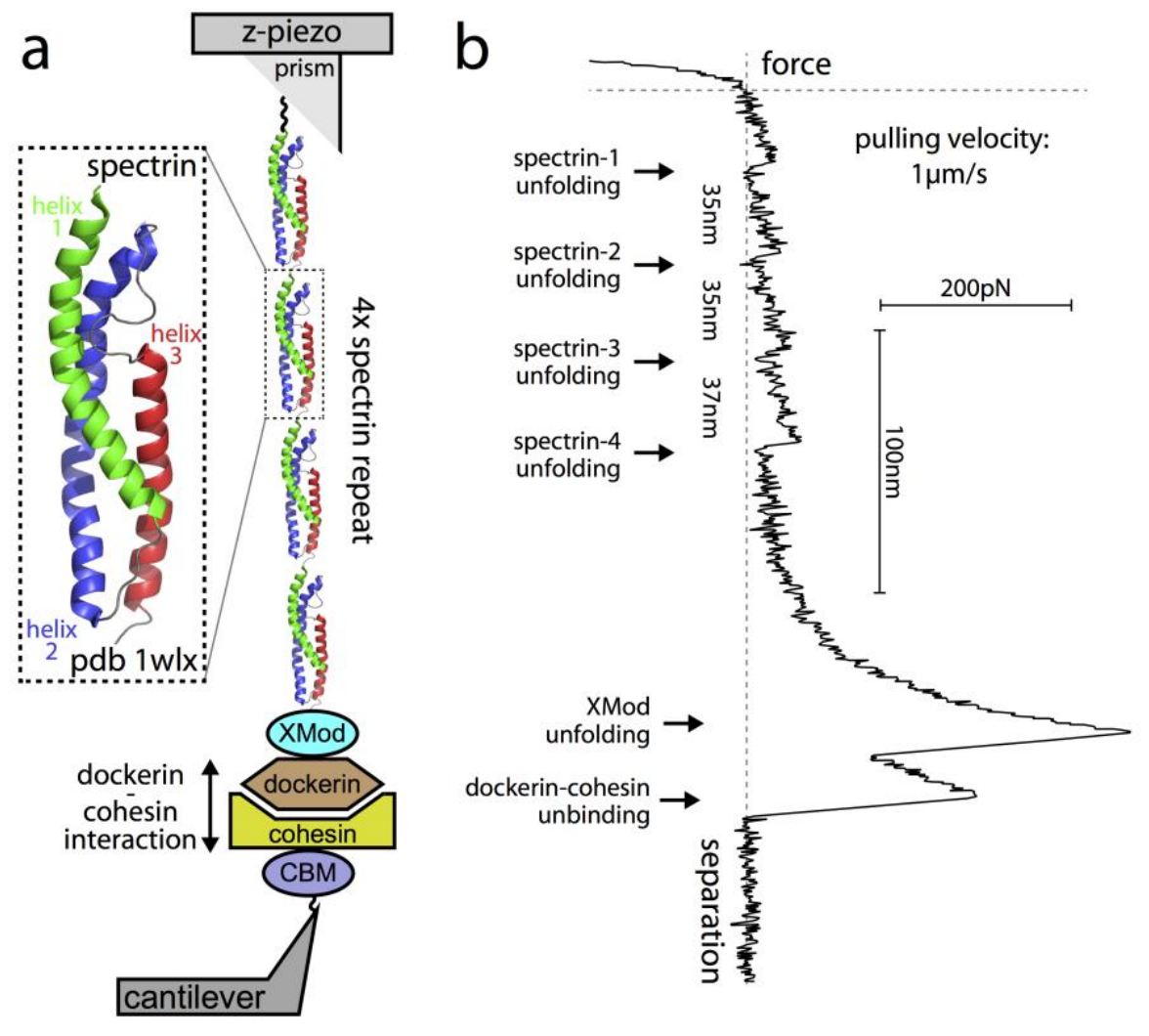

Figure 1. High-speed force spectroscopy (HS-FS) set-up for spectrin unfolding experiments. (a) Schematic illustration of the HS-FS set-up for spectrin unfolding: spectrin (PDB: 1WLX) concatamers of four repeats are immobilized to the glass substrate via a covalent bond. The spectrin concatamers are chimerized with Xmod and dockerin domains. On the HS-FS cantilever tip, cohesin, the binding partner of dockerin, was also immobilized covalently. (b) Forceextension curve showing four unfolding peaks. Since the cohesin-dockerin pair binds strongly $(>400 \mathrm{pN}$ unbinding force), the force distance curves were selected for the Xmod unfolding and cohesin-dockerin unbinding peak at a distance of $\sim 200 \mathrm{~nm}$ from the surface corresponding to the 
unfolding of the four spectrin repeats (with each spectrin repeat 129 amino acids long and an extension of $0.38 \mathrm{~nm}$ per amino acid, a full extension of $\sim 49 \mathrm{~nm}$ per spectrin repeat is expected). 


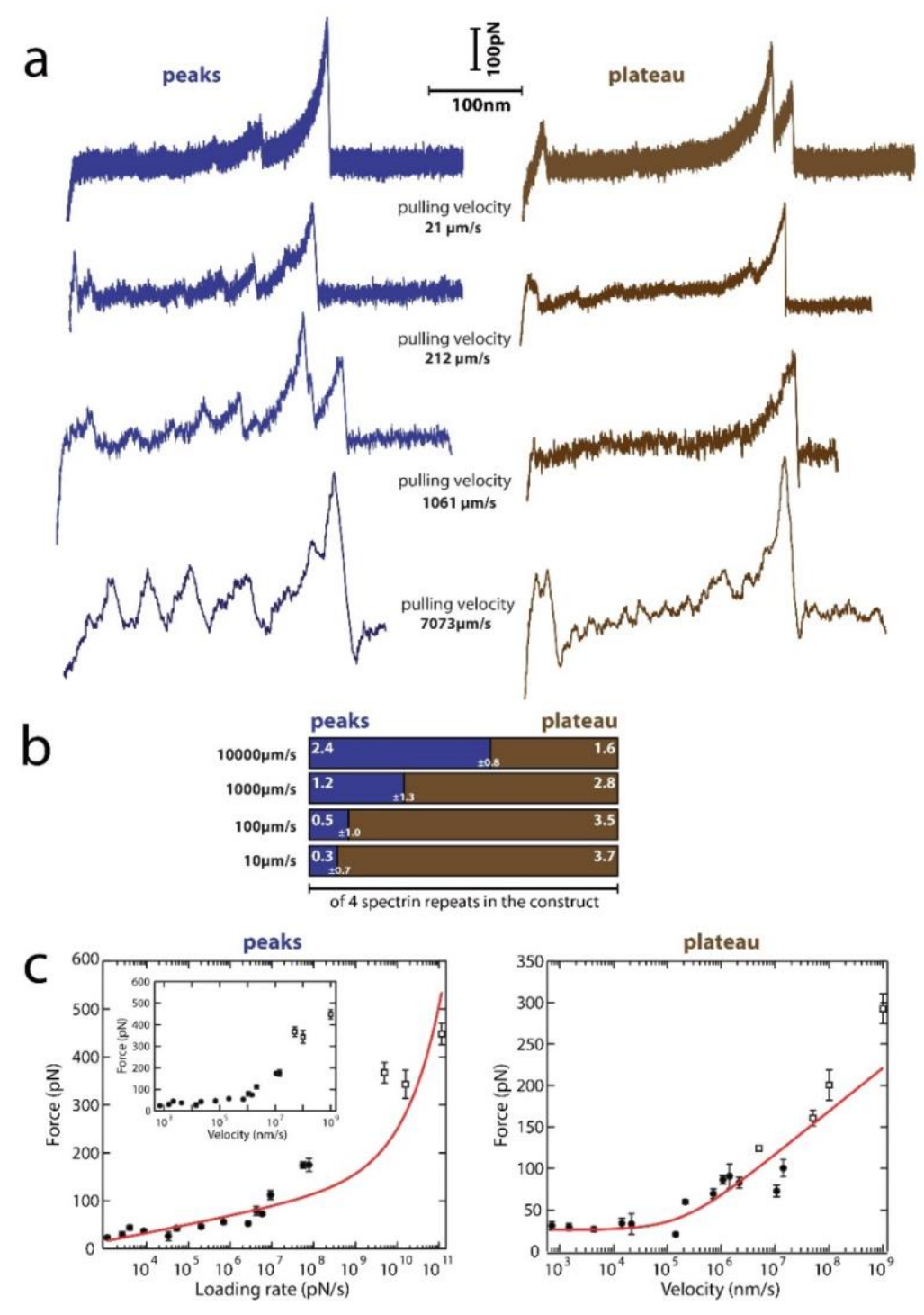

Figure 2. HS-FS spectrin unfolding and dynamic force spectra. (a) Force-extension curves acquired at different retraction velocities; $21 \mu \mathrm{m} / \mathrm{s}, 212 \mu \mathrm{m} / \mathrm{s}, 1061 \mu \mathrm{m} / \mathrm{s}$ and $7073 \mu \mathrm{m} / \mathrm{s}$. Forceextension curves with spectrin unfolding peaks (lefts) and spectrin unwinding force plateaus were observed at the different retraction velocities. (b) Average number of unfolding peaks and corresponding force plateau regions (of 4 possible spectrin repeats) detected per force-extension curve, at different retraction velocities; $10 \mu \mathrm{m} / \mathrm{s}, 100 \mu \mathrm{m} / \mathrm{s} 1000 \mu \mathrm{m} / \mathrm{s}$ and $10000 \mu \mathrm{m} / \mathrm{s}$. (c) Average unfolding peak forces (left) as a function of loading rate obtained by HS-FS experiment (filled markers) and SMDS simulation (open markers). Solid red line is the fit to the BSK model ${ }^{30}$ with fitting parameters of $D_{\mathrm{m}}=(3.4 \pm 0.6) \times 10^{6} \mathrm{~nm}^{2} / \mathrm{s}, x_{\beta}=0.53 \pm 0.03 \mathrm{~nm}$, and $\Delta G^{\ddagger}=18.4 \pm 0.4 k_{\mathrm{B}} T$ (see text). Inset: Average forces as a function of experimental pulling velocity. Average unwinding plateau forces (right) as a function of retraction velocity obtained by HS-FS experiment (filled markers) and MDS simulation (open markers). Solid red line is the fit to the FNdY model ${ }^{31}$ with fitting parameters of $x_{\mathrm{t}}=0.18 \pm 0.01 \mathrm{~nm}, f_{\mathrm{eq}}=26 \pm 2 \mathrm{pN}$, and $k_{\mathrm{NI}}^{0}=(6.7 \pm 1.0) \times 10^{6} \mathrm{~s}^{-1}$ (see text). 


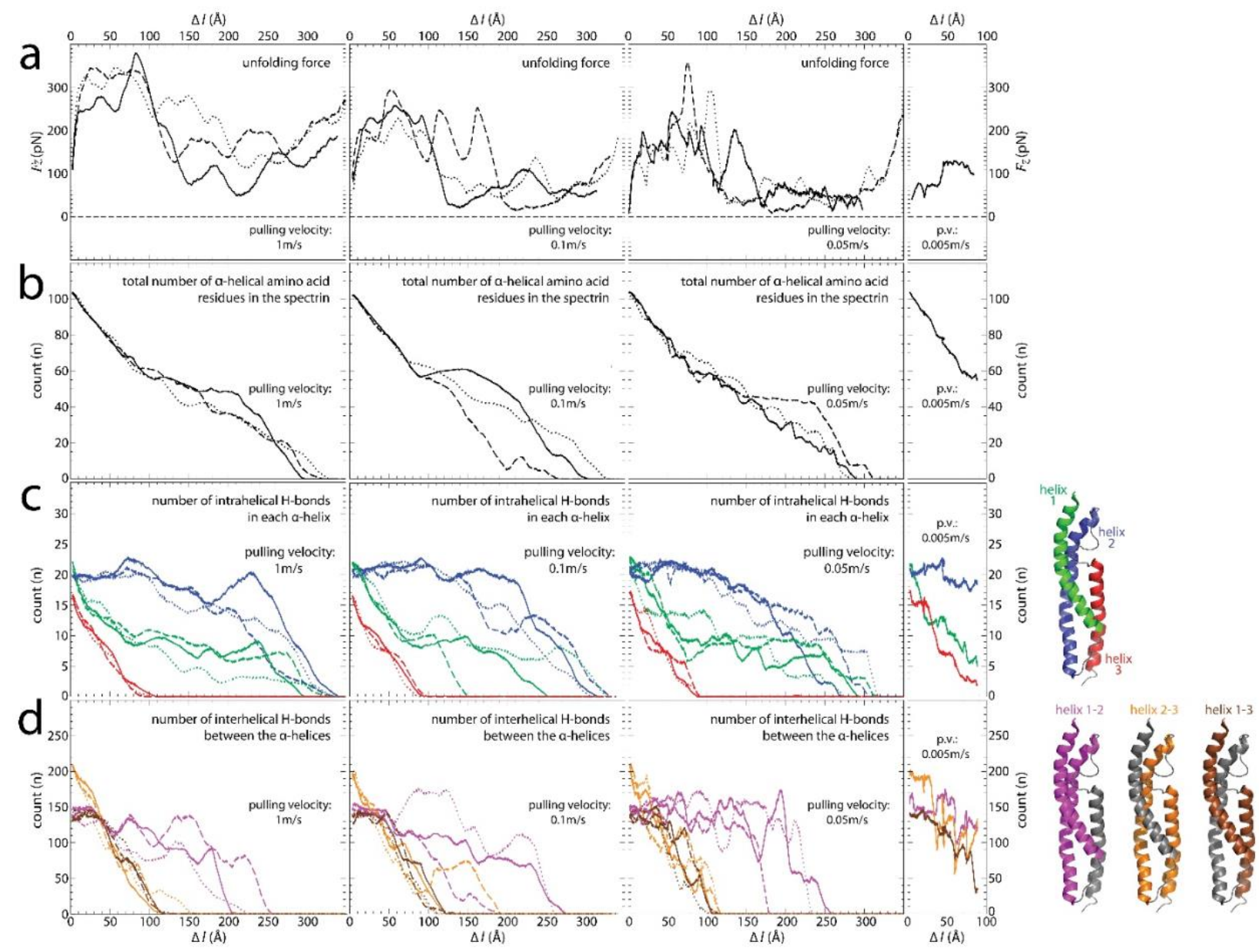

Figure 3. Steered molecular dynamics simulations (SMDS) pulling experiments of a single spectrin domain at $1.00 \mathrm{~m} \mathrm{~s}^{-1}\left(1 \mathrm{st}\right.$ column), at $0.10 \mathrm{~m} \mathrm{~s}^{-1}\left(2^{\text {nd }}\right.$ column), at $0.05 \mathrm{~m} \mathrm{~s}^{-1}\left(3^{\text {rd }}\right.$ column) and at $0.005 \mathrm{~m} \mathrm{~s}^{-1}\left(4^{\text {th }}\right.$ column). The profiles in each graph shown as a function of the extension, $\Delta l$, of the protein correspond to three independent simulations (by convention the first simulation is depicted as a solid line, the second as a dashed line and the third as a dotted line), with the exception of the simulation at $0.005 \mathrm{~ms}^{-1}$, where only one simulation to $\sim 100 \AA$ was performed. (a) Force versus extension, (b) total number of residues in the protein being in an $\alpha$ helical conformation, (c) number of intra-helical hydrogen bonds in the first (residues 1-36, green), second (43-78, blue) and third (100-129, red) $\alpha$-helices, and (d) number of interhelical contacts in the protein helix 1-2 (magenta), helix 2-3 (orange) and helix 1-3 (brown) are shown. For clarity, each panel is labeled according to the represented data and the pulling velocity. The structure on the right of (c) shows the spectrin repeat with the helices colored according to the helices unfolding trajectories depicted in (c), while the structures on the right of (d) show the spectrin repeat with helix pairs colored according to the helices interaction loss graphs shown in (d). 


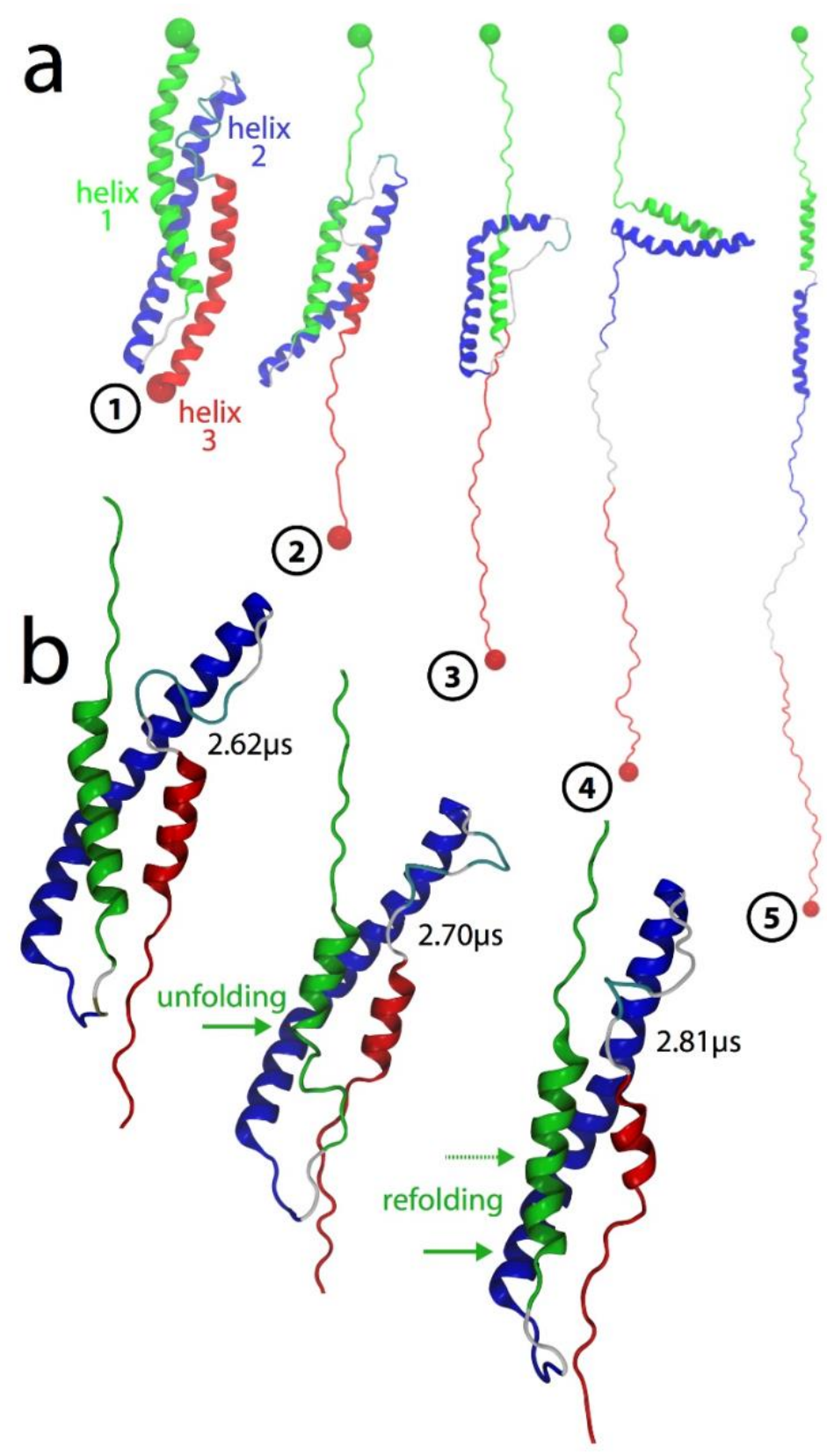

Figure 4. Series of snapshots of typical states observed during spectrin unfolding SMDS. (a) Initially, the spectrin bundle of three helices possesses an end-to-end distance of $\sim 70 \AA$ (N- to Cterminus, i.e. green sphere to red sphere) (state 1). Upon pulling force application, helices 1 and 3 start unwinding (state 2). Helix 3 is the shortest lived and the first to be completely unwound. Helix 2, now exposed to the pulling vector that is in opposite direction to its polarity, starts turning over (state 3). Helices 1 and 2 are in a zipper-like conformation, perpendicular to the force vector (state 4). Upon loss of interaction of helices 1 and 2, helix 1 unwinds, followed by helix 2 (state 5). The snapshots of the different states are not shown in scale. (b) Snapshots of the unfolding SMDS trajectory at $0.005 \mathrm{~ms}^{-1}$ pulling velocity. Recurrent helix unfolding (arrow in middle panel) and refolding is observed (helix between dashed and solid arrows).

ASSOCIATED CONTENT 


\section{Supporting Information.}

Supplementary Figures 1 and 2

Captions for Supplementary Videos 1 to 4

Other Supplementary Materials for this manuscript includes the following:

Supplementary Videos 1 to 4 

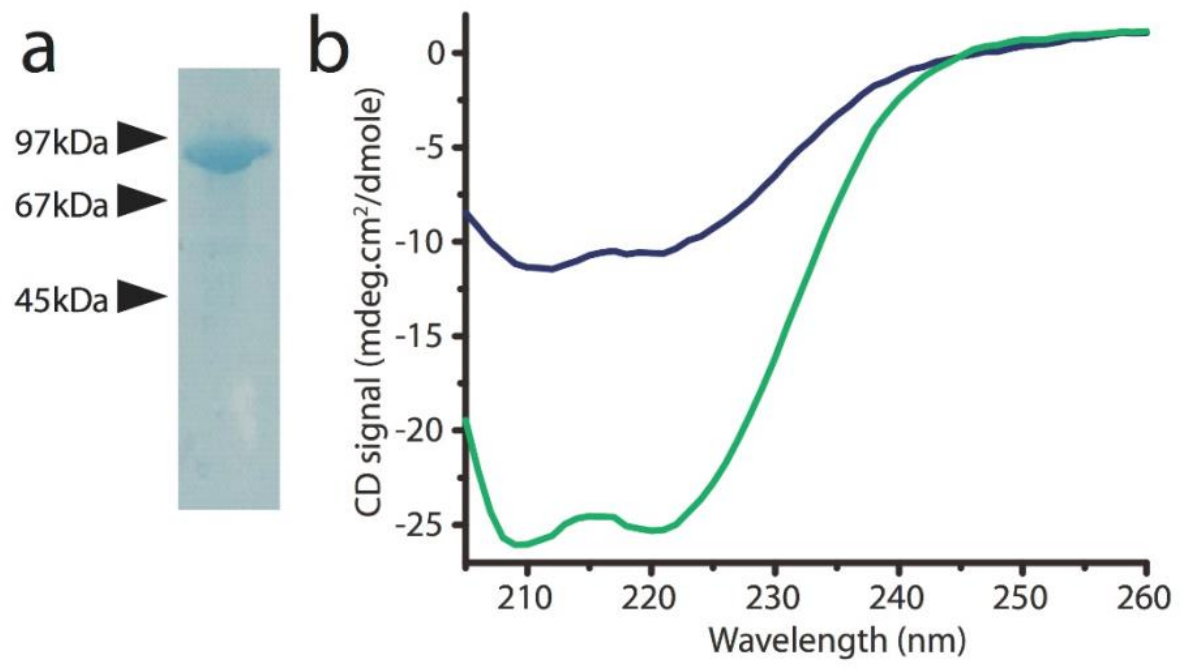

Supplementary Figure 1. Purification and circular dichroism analysis of spectrin-XModdockerin concatamer (a) SDS-PAGE of the purified spectrin(4 repeats)-XMod-dockerin concatamer. The purified fraction was analyzed by SDS-PAGE with $12 \%$ polyacrylamide gel and stained with coomassie brilliant blue. Arrowheads indicate molecular mass markers. The band of the purified fraction was estimated as $90 \mathrm{kDa}$, in good agreement with the expected molecular mass of the spectrin $(4$ repeats)-XMod-dockerin concatamer $(89.5 \mathrm{kDa})$. (b) Circular dichroism (CD) spectra analysis (spectrometer: J-810, Jasco) of the purified spectrin(4 repeats)XMod-dockerin concatamer. The concentration of the protein was adjusted to $100 \mu \mathrm{g} \mathrm{ml}^{-1}$ in $25 \mathrm{mM}$ Tris- $\mathrm{HCl}, \mathrm{pH} 7.5,75 \mathrm{mM} \mathrm{NaCl}, 2 \mathrm{mM} \mathrm{CaCl}_{2}$. The green and blue lines corresponding to the CD spectra of spectrin(4 reapeats)-XMod-dockerin and XMod-dockerin, respectively. 


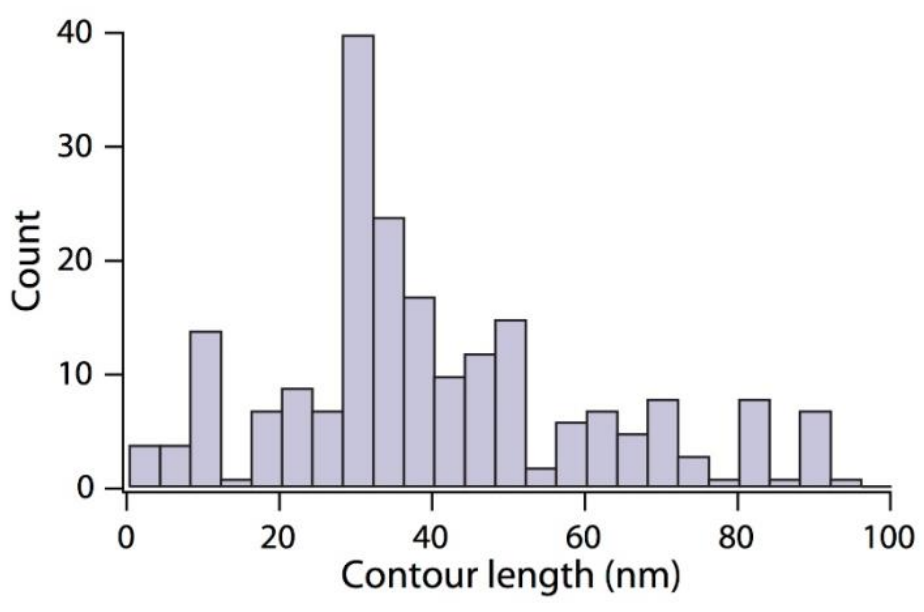

Supplementary Figure 2. Contour length analysis. Distribution of contour lengths between peaks from spectrin forced unfolding curves. Three main peaks are visible at $\sim 10 \mathrm{~nm}, \sim 35 \mathrm{~nm}$ and $\sim 50 \mathrm{~nm}$. Contour lengths larger than $50 \mathrm{~nm}$ were due to curves showing less than 4 peaks. Contour lengths longer than $100 \mathrm{~nm}$ were ignored from the analysis. 


\section{Captions for Supplementary Videos:}

Supplementary Video 1. SMDS spectrin unfolding at $1.00 \mathrm{~m} \mathrm{~s}^{\mathbf{1}}$. Helix-1 is shown in green, helix-2 in blue and helix-3 in red, respectively. The green sphere defines the average (fixed) position of the first amino acid (N-terminus) and the red sphere the average position of the last amino acid (C-terminus) during unfolding. The white dashed line denotes the end-to-end distance measured between the first and the last $\alpha$-carbon atoms. The number shown in the movie indicates the real time extension $(\AA)$ between the $\mathrm{N}$ - and C-termini during spectrin unfolding.

Supplementary Video 2. SMDS spectrin unfolding at $\mathbf{0 . 1 0} \mathrm{m} \mathrm{s}^{\mathbf{1}}$. Helix-1 is shown in green, helix-2 in blue and helix-3 in red, respectively. The green sphere defines the average (fixed) position of the first amino acid (N-terminus) and the red sphere the average position of the last amino acid (C-terminus) during unfolding. The white dashed line denotes the end-to-end distance measured between the first and the last $\alpha$-carbon atoms. The number shown in the movie indicates the real time extension $(\AA)$ between the $\mathrm{N}$ - and C-termini during spectrin unfolding.

Supplementary Video 3. SMDS spectrin unfolding at $\mathbf{0 . 0 5} \mathrm{m} \mathrm{s}^{-1}$. Helix-1 is shown in green, helix-2 in blue and helix-3 in red, respectively. The green sphere defines the average (fixed) position of the first amino acid (N-terminus) and the red sphere the average position of the last amino acid (C-terminus) during unfolding. The white dashed line denotes the end-to-end distance measured between the first and the last $\alpha$-carbon atoms. The number shown in the movie indicates the real time extension $(\AA)$ between the $\mathrm{N}$ - and C-termini during spectrin unfolding.

Supplementary Video 4. SMDS spectrin unfolding at $0.005 \mathrm{~m} \mathrm{~s}^{\mathbf{- 1}}$. Helix-1 is shown in green, helix-2 in blue and helix-3 in red, respectively. The green sphere defines the average (fixed) position of the first amino acid (N-terminus) and the red sphere the average position of the last amino acid (C-terminus) during unfolding. The white dashed line denotes the end-to-end distance measured between the first and the last $\alpha$-carbon atoms. The number shown in the movie indicates the real time extension $(\AA)$ between the $\mathrm{N}$ - and C-termini during spectrin unfolding. 


\title{
AUTHOR INFORMATION
}

Corresponding Author

Simon Scheuring*

Email: sis2019@med.cornell.edu

Tel; + (1) 646-962-2565

Department of Anesthesiology, Weill Cornell Medical College, 1300 York Ave, New York, NY 10065, USA;

\section{AUTHOR CONTRIBUTIONS}

Hirohide Takahashi ${ }^{\dagger}$ and Felix Rico ${ }^{\dagger}$ contributed equally.

\section{FUNDING SOURCES}

ANR grants (ANR-11-IDEX-0001-02: A*MIDEX and ANR-15-CE11-0007-01: BioHSFS) and European Research Council (ERC) Grant (\#310080; MEM-STRUCT-AFM).

\section{ACKNOWLEDGMENT}

We thank Michael Nash and Wolfgang Ott for sharing the XMod-dockerin-III and cohesin-III plasmids and for technical assistance. We are also grateful to Drs. Kunio Takeyasu and Masahiro Kumeta for providing the spectrin DNA plasmid. This work was supported by ANR grants (ANR-11-IDEX-0001-02: A*MIDEX and ANR-15-CE11-0007-01: BioHSFS) and a European Research Council (ERC) Grant (\#310080; MEM-STRUCT-AFM).

\author{
ABBREVIATIONS \\ AFM; Atomic force microscopy \\ FS; Force spectroscopy \\ SMDS; Steered molecular dynamics simulations \\ HS-FS; High-speed force spectroscopy \\ $\mathrm{CD}$; Circular dichroism \\ MD; Molecular dynamics
}




\section{REFERENCES}

1. Jaalouk, D. E.; Lammerding, J. Nature Reviews Molecular Cell Biology 2009, 10, 63-73.

2. Elosegui-Artola, A.; Andreu, I.; Beedle, A. E. M.; Lezamiz, A.; Uroz, M.; Kosmalska, A. J.; Oria, R.; Kechagia, J. Z.; Rico-Lastres, P.; Le Roux, A. L.; Shanahan, C. M.; Trepat, X.; Navajas, D.; Garcia-Manyes, S.; Roca-Cusachs, P. Cell 2017, 171, (6), 1397-1410 e14.

3. Bennett, V.; Gilligan, D. M. Annu Rev Cell Biol 1993, 9, 27-66.

4. $\quad$ Bennett, V.; Baines, A. J. Physiol Rev 2001, 81, (3), 1353-92.

5. $\quad$ Bennett, V.; Stenbuck, P. J. Nature 1979, 280, (5722), 468-73.

6. $\quad$ Xu, K.; Zhong, G.; Zhuang, X. Science 2013, 339, (6118), 452-6.

7. $\quad$ Speicher, D. W.; Marchesi, V. T. Nature 1984, 311, (5982), 177-80.

8. Johnson, C. P.; Tang, H. Y.; Carag, C.; Speicher, D. W.; Discher, D. E. Science 2007, 317, (5838), 663-6.

9. $\quad$ Broderick, M. J.; Winder, S. J. Adv Protein Chem 2005, 70, 203-46.

10. Nicolas, A.; Delalande, O.; Hubert, J. F.; Le Rumeur, E. J Struct Biol 2014, 186, (3), 392401.

11. Liem, R. K. Cold Spring Harb Perspect Biol 2016, 8, (10).

12. Yan, Y.; Winograd, E.; Viel, A.; Cronin, T.; Harrison, S. C.; Branton, D. Science 1993, 262, (5142), 2027-30.

13. Rief, M.; Gautel, M.; Oesterhelt, F.; Fernandez, J. M.; Gaub, H. E. Science 1997, 276, (5315), 1109-12.

14. Florin, E. L.; Moy, V. T.; Gaub, H. E. Science 1994, 264, (5157), 415-7.

15. Rief, M.; Pascual, J.; Saraste, M.; Gaub, H. E. J Mol Biol 1999, 286, (2), 553-61.

16. Lenne, P. F.; Raae, A. J.; Altmann, S. M.; Saraste, M.; Horber, J. K. FEBS Lett 2000, 476, (3), 124-8.

17. Law, R.; Carl, P.; Harper, S.; Dalhaimer, P.; Speicher, D. W.; Discher, D. E. Biophys $J$ 2003, 84, (1), 533-44.

18. Randles, L. G.; Rounsevell, R. W.; Clarke, J. Biophys $J$ 2007, 92, (2), 571-7.

19. Altmann, S. M.; Grunberg, R. G.; Lenne, P. F.; Ylanne, J.; Raae, A.; Herbert, K.; Saraste, M.; Nilges, M.; Horber, J. K. Structure 2002, 10, (8), 1085-96.

20. Rico, F.; Gonzalez, L.; Casuso, I.; Puig-Vidal, M.; Scheuring, S. Science 2013, 342, (6159), 741-3.

21. Soncini, M.; Vesentini, S.; Ruffoni, D.; Orsi, M.; Deriu, M. A.; Redaelli, A. Biomech Model Mechanobiol 2007, 6, (6), 399-407.

22. Isralewitz, B.; Gao, M.; Schulten, K. Curr Opin Struct Biol 2001, 11, (2), 224-30.

23. Sotomayor, M.; Schulten, K. Science 2007, 316, (5828), 1144-1148.

24. Ortiz, V.; Nielsen, S. O.; Klein, M. L.; Discher, D. E. J Mol Biol 2005, 349, (3), 638-47.

25. Mucksch, C.; Urbassek, H. M. J Chem Theory Comput 2016, 12, (3), 1380-4.

26. Djinovic-Carugo, K.; Gautel, M.; Ylanne, J.; Young, P. FEBS Lett 2002, 513, (1), 119 -

23.

27. Schoeler, C.; Malinowska, K. H.; Bernardi, R. C.; Milles, L. F.; Jobst, M. A.; Durner, E.;

Ott, W.; Fried, D. B.; Bayer, E. A.; Schulten, K.; Gaub, H. E.; Nash, M. A. Nat Commun 2014, 5,5635 .

28. Evans, E.; Ritchie, K. Biophys J 1997, 72, (4), 1541-55.

29. Merkel, R.; Nassoy, P.; Leung, A.; Ritchie, K.; Evans, E. Nature 1999, 397, (6714), 5053. 
30. Bullerjahn, J. T.; Sturm, S.; Kroy, K. Nat Commun 2014, 5, 4463.

31. Friddle, R. W.; Noy, A.; De Yoreo, J. J. Proceedings of the National Academy of Sciences of the United States of America 2012, 109, (34), 13573-8.

32. Rief, M.; Fernandez, J. M.; Gaub, H. E. Physical Review Letters 1998, 81, (21), 47644767.

33. Sader, J. E.; Chon, J. W. M.; Mulvaney, P. Review of Scientific Instruments 1999, 70, (10), 3967-3969.

34. Hutter, J. L.; Bechhoefer, J. Rev. Sci. Instrum. 1993, 64, 1868.

35. Higgins, M. J.; Proksch, R.; Sader, J. E.; Polcik, M.; Endoo, S. M.; Cleveland, J. P.; Jarvis, S. P. Review of Scientific Instruments 2006, 77, (1), 013701.

36. Schillers, H.; Rianna, C.; Schape, J.; Luque, T.; Doschke, H.; Walte, M.; Uriarte, J. J.; Campillo, N.; Michanetzis, G. P. A.; Bobrowska, J.; Dumitru, A.; Herruzo, E. T.; Bovio, S.; Parot, P.; Galluzzi, M.; Podesta, A.; Puricelli, L.; Scheuring, S.; Missirlis, Y.; Garcia, R.; Odorico, M.; Teulon, J. M.; Lafont, F.; Lekka, M.; Rico, F.; Rigato, A.; Pellequer, J. L.; Oberleithner, H.; Navajas, D.; Radmacher, M. Sci Rep 2017, 7, (1), 5117.

37. Phillips, J. C.; Braun, R.; Wang, W.; Gumbart, J.; Tajkhorshid, E.; Villa, E.; Chipot, C.; Skeel, R. D.; Kale, L.; Schulten, K. J Comput Chem 2005, 26, (16), 1781-802.

38. MacKerell, A. D.; Bashford, D.; Bellott, M.; Dunbrack, R. L.; Evanseck, J. D.; Field, M. J.; Fischer, S.; Gao, J.; Guo, H.; Ha, S.; Joseph-McCarthy, D.; Kuchnir, L.; Kuczera, K.; Lau, F. T.; Mattos, C.; Michnick, S.; Ngo, T.; Nguyen, D. T.; Prodhom, B.; Reiher, W. E.; Roux, B.; Schlenkrich, M.; Smith, J. C.; Stote, R.; Straub, J.; Watanabe, M.; Wiorkiewicz-Kuczera, J.; Yin, D.; Karplus, M. J Phys Chem B 1998, 102, (18), 3586-616.

39. Tuckerman, M.; Berne, B. J.; Martyna, G. J. J. Phys. Chem. B 1992, 97, (3), 1990-2001.

40. Andersen, H. C. J. Comput. Phys. 1983, 52, (1), 24-34.

41. Miyamoto, S.; Kollman, P. A. J. Comput. Chem. 1992, 18, (8), 952-962.

42. Feller, S. E.; Zhang, Y.; Pastor, R. W.; Brooks, B. R. J. Chem. Phys. 1995, 103, 4613.

43. Humphrey, W.; Dalke, A.; Schulten, K. J Mol Graph 1996, 14, (1), 33-8, 27-8. 


\section{Supplementary Materials for}

\section{$\alpha$-Helix Unwinding as Force Buffer in Spectrins}

Hirohide Takahashi ${ }^{1,2,3 \ddagger}$, Felix Rico ${ }^{1 \ddagger}$, Christophe Chipot $^{4} \&$ Simon Scheuring ${ }^{1,2,3^{*}}$

${ }^{1}$ U1006 INSERM, Université Aix-Marseille, Parc Scientifique et Technologique de Luminy, 163 avenue de Luminy, 13009 Marseille, France

${ }^{2}$ Department of Anesthesiology, Weill Cornell Medical College, 1300 York Ave, New York, NY 10065, USA

${ }^{3}$ Departments of Physiology and Biophysics, Weill Cornell Medical College, 1300 York Ave, New York, NY 10065, USA

${ }^{4}$ Laboratoire International Associé Centre National de la Recherche Scientifique et University of Illinois at UrbanaChampaign, UMR 7565, Université de Lorraine, B.P. 70239, 54506 Vandœuvre-lès-Nancy cedex, France and Department of Physics, University of Illinois at Urbana-Champaign, 1110 West Green Street, Urbana, Illinois 61801, USA

*Equal author contribution.

* Correspondence to: S. Scheuring, Email: sis2019@med.cornell.edu

This file includes:

Supplementary Figures 1 and 2

Captions for Supplementary Videos 1 to 4

Other Supplementary Materials for this manuscript includes the following:

Supplementary Videos 1 to 4 


\section{Supporting Information.}

Supplementary Figures 1 and 2

Captions for Supplementary Videos 1 to 4

Other Supplementary Materials for this manuscript includes the following:

Supplementary

Videos

1

to 


\section{Supplementary figures:}

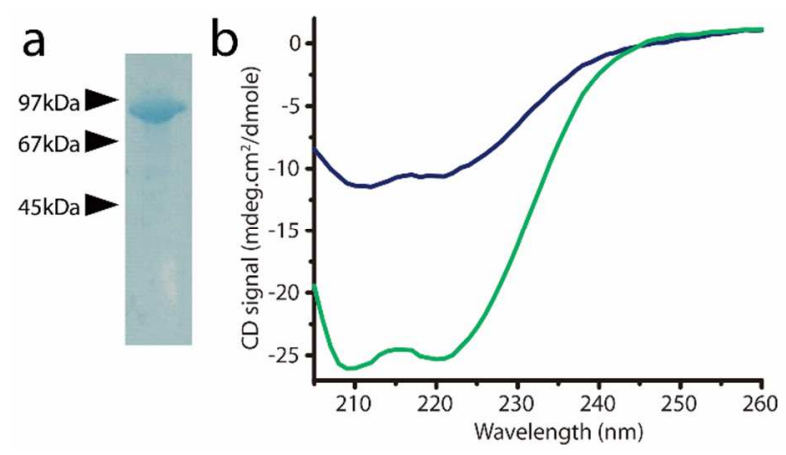

Supplementary Figure 1. Purification and circular dichroism analysis of spectrin-XModdockerin concatamer. (a) SDS-PAGE of the purified spectrin(4 repeats)-XMod-dockerin concatamer. The purified fraction was analyzed by SDS-PAGE with $12 \%$ polyacrylamide gel and stained with coomassie brilliant blue. Arrowheads indicate molecular mass markers. The band of the purified fraction was estimated as $90 \mathrm{kDa}$, in good agreement with the expected molecular mass of the spectrin(4 repeats)-XMod-dockerin concatamer $(89.5 \mathrm{kDa})$. (b) Circular dichroism (CD) spectra analysis (spectrometer: J-810, Jasco) of the purified spectrin(4 repeats)XMod-dockerin concatamer. The concentration of the protein was adjusted to $100 \mu \mathrm{g} \mathrm{ml}^{-1}$ in $25 \mathrm{mM}$ Tris- $\mathrm{HCl}, \mathrm{pH} 7.5,75 \mathrm{mM} \mathrm{NaCl}, 2 \mathrm{mM} \mathrm{CaCl}_{2}$. The green and blue lines corresponding to the CD spectra of spectrin(4 reapeats)-XMod-dockerin and XMod-dockerin, respectively. 


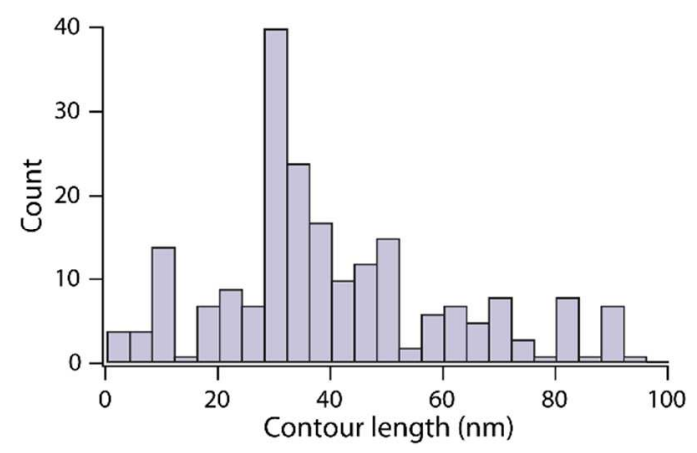

Supplementary Figure 2. Contour length analysis. Distribution of contour lengths between peaks from spectrin forced unfolding curves. Three main peaks are visible at $\sim 10 \mathrm{~nm}, \sim 35 \mathrm{~nm}$ and $\sim 50 \mathrm{~nm}$. Contour lengths larger than $50 \mathrm{~nm}$ were due to curves showing less than 4 peaks. Contour lengths longer than $100 \mathrm{~nm}$ were ignored from the analysis. 


\section{Captions for Supplementary Videos:}

Supplementary Video 1. SMDS spectrin unfolding at $1.00 \mathrm{~m} \mathrm{~s}^{-1}$. Helix-1 is shown in green, helix-2 in blue and helix-3 in red, respectively. The green sphere defines the average (fixed) position of the first amino acid (N-terminus) and the red sphere the average position of the last amino acid (C-terminus) during unfolding. The white dashed line denotes the end-to-end distance measured between the first and the last $\alpha$-carbon atoms. The number shown in the movie indicates the real time extension $(\AA)$ between the $\mathrm{N}$ - and C-termini during spectrin unfolding.

Supplementary Video 2. SMDS spectrin unfolding at $0.10 \mathrm{~m} \mathrm{~s}^{-1}$. Helix-1 is shown in green, helix-2 in blue and helix-3 in red, respectively. The green sphere defines the average (fixed) position of the first amino acid (N-terminus) and the red sphere the average position of the last amino acid (C-terminus) during unfolding. The white dashed line denotes the end-to-end distance measured between the first and the last $\alpha$-carbon atoms. The number shown in the movie indicates the real time extension $(\AA)$ between the $\mathrm{N}$ - and C-termini during spectrin unfolding.

Supplementary Video 3. SMDS spectrin unfolding at $0.05 \mathrm{~m} \mathrm{~s}^{-1}$. Helix-1 is shown in green, helix-2 in blue and helix-3 in red, respectively. The green sphere defines the average (fixed) position of the first amino acid (N-terminus) and the red sphere the average position of the last amino acid (C-terminus) during unfolding. The white dashed line denotes the end-to-end distance measured between the first and the last $\alpha$-carbon atoms. The number shown in the movie indicates the real time extension $(\AA)$ between the $\mathrm{N}$ - and C-termini during spectrin unfolding.

Supplementary Video 4. SMDS spectrin unfolding at $0.005 \mathrm{~m} \mathrm{~s} \mathbf{s}^{-1}$. Helix-1 is shown in green, helix-2 in blue and helix-3 in red, respectively. The green sphere defines the average (fixed) position of the first amino acid (N-terminus) and the red sphere the average position of the last amino acid (C-terminus) during unfolding. The white dashed line denotes the end-to-end distance measured between the first and the last $\alpha$-carbon atoms. The number shown in the movie indicates the real time extension $(\AA)$ between the $\mathrm{N}$ - and C-termini during spectrin unfolding. 
